\title{
Recognize Flexible Malevolent Web pages in Real Time
}

\author{
Bhargav Kakani, Surendra Reddy
}

\begin{abstract}
Mobile precise internet web sites dissent drastically from their computer laptop equivalents in cloth, format and functionality. Sooner or later, present techniques to sight detrimental net internet internet sites rectangular movement now not probably to determine for such webpages. During this paper, we often typically have a propensity to format and exercising paintings over, a mechanism that distinguishes amongst terrible and benign mobile net net web sites. Activity over makes this energy of will supported normal picks of a net internet web page beginning with the quantity of iframes to the life of identified dishonourable cellular mobile cellphone numbers. First, we have a tendency to via attempting out show the requirement for mobile information strategies so installation a spread of new regular options that very correlate with cellular malicious pages. We will be predisposed to then use work over to a dataset of over 350,000 famous benign similarly to volatile cellular webpages and show 90 th accuracy in splendor. In addition, we frequently normally normally have a tendency to discover, end up aware of and furthermore document choice of websites incomprehensible through Google Safe Surfing and furthermore Virus Total, however decided through art work over. Lastly, we will be inclined to growth a web browser extension victimization undertaking over to comfortable customers from damaging mobile internet web sites in length. In doing consequently, we provide the number one everyday assessment technique to view volatile cellular webpages.
\end{abstract}

\section{INTRODUCTION}

On the internet casual organizations (OSNs) that collaborate virtual cash fill in as an appealing diploma for various industrial organisation company enterprise company sports activities, in which on the internet, intuitive innovation is amongst one of the most colourful ones. Specifically, a consumer, who is usually spoken with thru her OSN account, can get make up in the form of digital coins with the beneficial useful aid of taking detail on-line development wearing sports sports imposed via the use of organisation elements. She may additionally moreover want to really and then have the potential to utilize such remunerate mainly programs, for example, net based simply certainly looking for, converting it to distinct humans, similarly to now not preserving up in competition to looking for and promoting it for actual cash.Such virtual-coins empowered on the internet innovation display empowers massive initiative, factors guide budgetary boosts to complete clients, what's extra, inside the period in-between rules the corporations in amongst organisation factors and economic centers. Therefore, this model has indicated exquisite guarantee and improved extremely good control rapid.In any shape of scenario, it encounters an vital chance: enemies can control a massive style of documents, either via manner of enlisting new statistics in any other case all all over again negotiating gift information, to take a fee of interest within the at the net. It consequently becomes of important importance to discover money owed controlled through opponents in at the internet merchandising sports.In the adhering to conversations, we communicate with such money owed as unfavourable money owed.

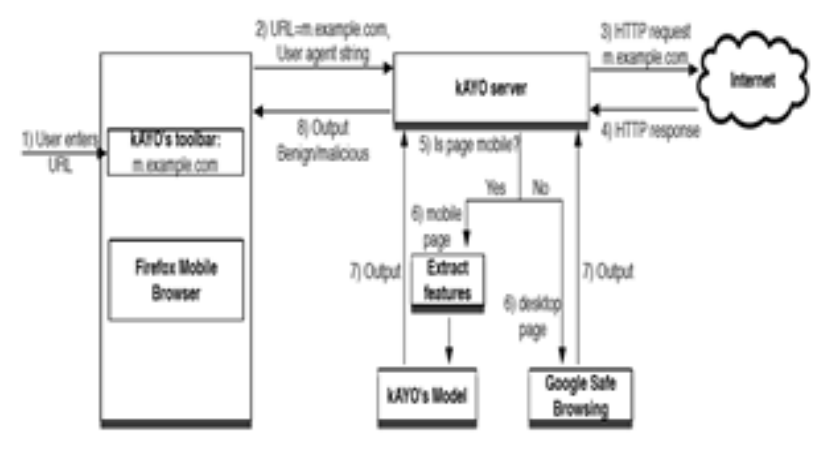

The effective discovery of lousy bills permits every OSNs and provider entities to take reduce price sports activities sports sports activities sports sports activities sports activities which embody prohibiting those debts or decreasing the bills as malicious debts. The reliable detection of malicious debts allows every OSNs similarly to agency entities to take mitigation sports sportssportssports activities sports which includes outlawing the ones debts or decreasing the possibility to award those payments. Nevertheless, making an powerful discovery technique is confronted with more than one massive boundaries. Initially, aggressors do no longer need to create malicious internet content material fabric cloth cloth (e.G., phishing Links and moreover risky executables) to launch a success attacks. Comparatively, fighters can effectively do assaults via the usage of in reality clicking links used by organization entities or sharing the benign internet content cloth material material cloth that is on the begin disbursed through company employer partners. These moves themselves do no longer perceivably set aside from benign money owed. Second, a fulfillment moves do no longer require to depend on social Structures(e.G., "adhering to" or "particular buddy" dating in preferred social media networks). To be

Revised Manuscript Received on September 10, 2019.

BhargavKakani *, CSE department, Newtons Institute of Engineering, Macherla, A.P. India.

Dr. Surendra Reddy, CSE department, Newtons Institute of Engineering, Macherla, A.P. India. 
extra unique, maintaining energetic social frameworks does now not earnings to assailants, which is basically outstanding from terrific actions which consist of spammers in at the net socials media. These demanding situations make the detection of such volatile OSN money owed basically precise from the invention of conventional assaults which encompass spamming and moreover phishing. Therefore, it's miles quite difficult to cope with present strategies to understand spamming and moreover phishing debts. Existing RMMs for cloud computing collect responses from clients similarly to combine them to get tune statistics for the cycle. It attempts to break up among honest feedback from unfair responses supplied via manner of manner of the customers concerning the performance of the cycle. It conjointly distinguishes among faults within the physical networks and consequently the planned sports activities activitiesactivities sports sports activities sports sports of cycle that reason disruption inside the bodily network. Therefore, errors (which place device presumed to be beyond the tracking of the $\mathrm{CP}$ ) do not effect music records of cycle Sun et.Al. Projected a multi-faceted rely on monitoring model with the reason to interrupt up in amongst proper and furthermore unjust Feedbacks regarding the cloud agencies. Wanget.Al conjointly projected a multifaceted name control model that lets in the humans to gauge the Cloud issuer' exploitation numerous alternatives. Sidhut.Al. Forecasted a consider assessment of the Cloud businesses sustained the infraction of agreements delineated inside the answer diploma settlement. Macias et.Al predicted a mechanism to isolate unfair and moreover terrible bear in mind fundcloud laptop. Maccaset. Al. Expected a plan on call control that decreases the impact of tool failing at the selection of the cloud businesses. In this paper, we frequently normally will be inclined to signify a strong RMM within the federate cloud with intention multitenancy. In a very multi-tenant cloud, a consumer is based definitely upon at the $\mathrm{CP}$ for sincere carbon monoxidetenants.In this paper we will be inclined to suggest a very specific call monitoring tool that motivates the cycles/2d to assign smart co-tenants to a sincere man or woman. In this paper we frequently will be predisposed to suggest a mechanism that urges cycles/2nd to record suitable responses concerning the customers. Quickly, our RMM capabilities as follows: First, each CP distinguishes malicious clients from clever people and it need to designate assets to them particular the ubsequent holds: It want to now not permit any form of malicious individual to end up a cotenant of a honest individual. It have to allow unfavorable Following, the cycles/2d percentage statistics traumatic multitenancy's.
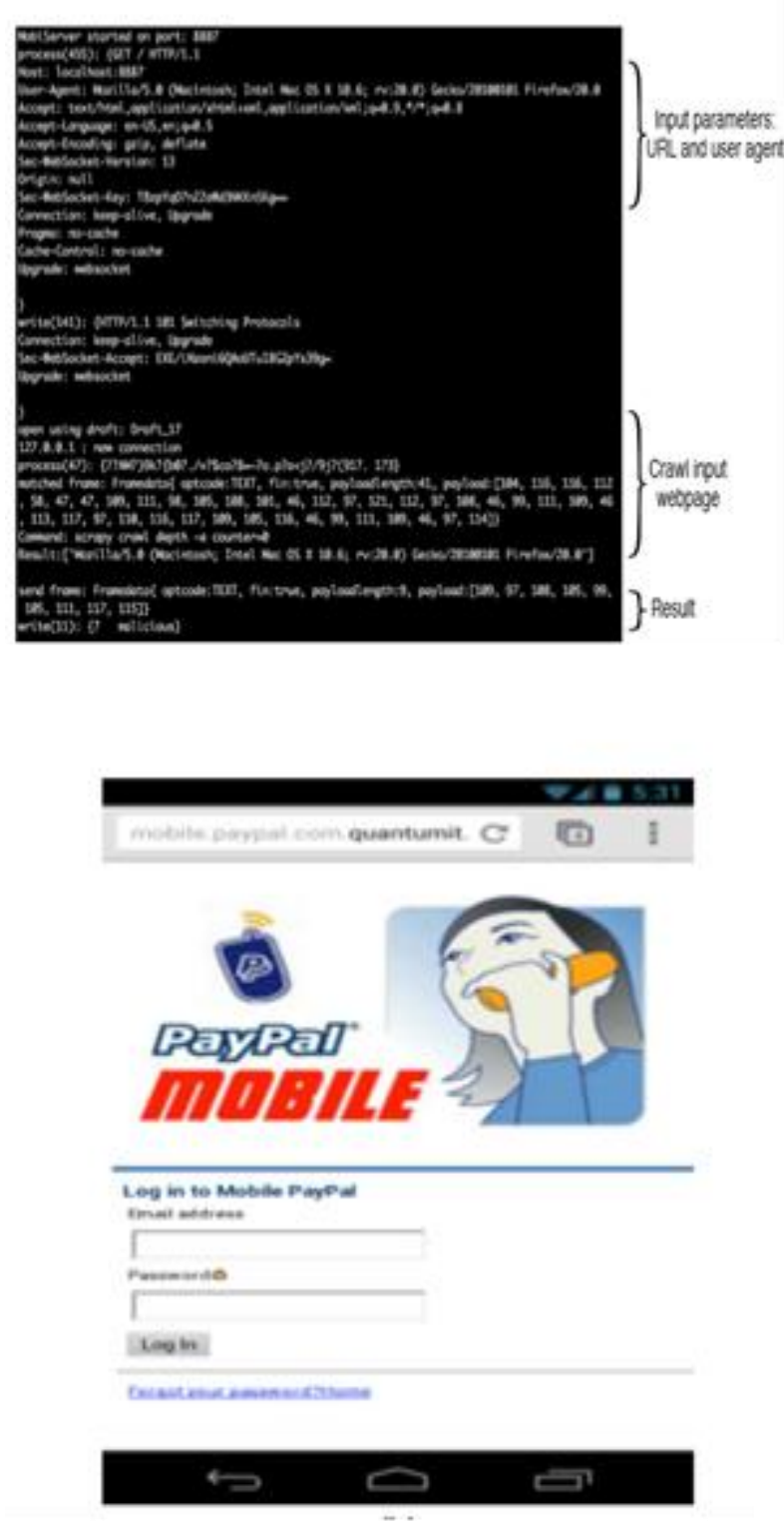

\section{RELATED FUNCTION}

Since online informal groups play an developing vital machine in each virtual and moreover corporation global, identifying malignant clients in OSNs takes area to tremendous importance. Countless acknowledgment strategies had been as a end result recommended [1], [2], [3], [4], [5], [6], [7], [8] Considering the popularity of spammers in OSNs, the ones strategies awesome center spherical determining money owed that supply pernicious compound. A spamming assault can be taken into consideration as a information waft started out with an assailant, with a development of malignant files, in the long run to an unfortunate casualty account. Even with the numerous shape of these strategies, they particularly use fractional or every taken into consideration one in each of three hotspots for identification together with I) the substance of the direct mail message, ii) the tool foundation that has the pernicious facts (e.G., phishing net content cloth 
cloth or however endeavors), and iii) the social form amongst malignant files and wounded non-public files. For instance, Gao et al. [9] intended a way to find out fights of pernicious information with the useful resource of way of the usage of manner of bunching money owed that deliver out messages with similar cloth. Lee et al. [10] conceived a manner to initially have a have a have a observe HTTP redirection chains commenced out from URLs set up in an OSN message, at that element built messages that brought approximately internet net website on-line on line pages promoted in a comparable net server, final however now not least made use of the server recognition to substantially identified vengeful information. Yang et al. [11] removed a chart from the "coming with" partnership of twitter records and later created perniciousness score the use of the inferred chart; Wu et al. [7] proposed a social spammer in addition to junk mail message codetection technique primarily based completely mostly on the posting relationships among clients what's even greater, messages, and furthermore made use of the partnership amongst customer and furthermore message to beautify the execution of each social spammer exploration. Contrasted with present day-day techniques on spotting spamming bills in OSNs, it is regarded with logonew problems to understand malevolent money owed that partake in online development physical sports. To start with, no longer as an opportunity much like spamming debts, those files now not one or the severa one in every of a kind rely on spamming messages nor require negative device foundations to dispatch assaults. Second, social systems are maximum absolutely now not crucial. Along those traces, none of gift strategies relates to identifying poisonous documents in on-line innovation workout bodily sports activities. O cope with the brand-new problems, our technique identifies terrible debts through finding out every not unusual sports activities sports sportssports activities activities of a document what is even greater, its cash associated sports activities sports activities sports. Distinct sly physical sports activities in financial exchanges has further pulled in huge research endeavors [13], [14] For example, Olszewski et al [15] talked with the client account data in 2-dimensional room of the Self-Organizing Map lattice, and furthermore encouraged a reputation method primarily based totally on seize 22 situation type double characterization estimation to deal with problems of Visa misrepresentation and furthermore media communications extortion. Lin et al. [16] located the importance of misrepresentation variables finished in spending plan report misstatement acknowledgment, and researched the exceptional order charges of three estimations collectively with Logistic Regression, Decision Trees, and moreover Artificial Neural Networks. Throckmorton et al. [17] advised a business enterprise enterprise coins associated misstatement place technique due to blended highlights of monetary numbers, semantic conduct, similarly to nonverbal vocal. Contrasted with the centered cash associated extortion exploration problems, account practices of collecting furthermore, using the digital cash in on the internet innovation exercise physical sports sports are definitely fantastic with ordinary economic systems thinking about that they do not in reality encompass budgetary exercise exercise sports but further arranging and online development sports activities sports. To abridge, our undertaking anticipates to address one extra hassle because of the logo-new sample of walking with online casual organizations furthermore, budgetary sporting activities. ProGuard consists of logo-new capability of intertwining highlights from every structures control similarly to cash associated detail of perspectives for exploration. In an OSN that integrates monetary responsibilities, an OSN account is usually related to represent each on-line banking further to digital foreign exchange. Number 1 offers such an instance, wherein a QQ account, the maximum preferred OSN account of Tencent, is related to an digital banking account for actual cash and a make up digital forex (i.E., Q coin). A client usually proper now down payments real overseas cash proper into her digital banking account; she is probably capable of recharge her digital forex account from her economic account. By taking element on the net vending sports activities sports sports, a purchaser can also reenergize her on-line cash account thru amassing blessings from the vending sports activities sports. A purchaser can burn up from his debts in common strategies. First, she may be capable to utilize actual or virtual foreign exchange to shop for each right and furthermore on line merchandise (i.E., on line shopping for). Second, she can be capable of transfer each real and digital distant places coins to every different character with the useful resource of sending out gadgets.Figure 2 gives the ordinary virtual coins circulate at the same time as malicious debts participate in at the net selling sports. The flow into is made from 3 degrees which includes i) gathering, ii) multi-layer transferring, and iii) laundering the virtual forex. In preliminary diploma, an opponent controls a tough and rapid of money owed to participate in online corporation vending sports activities and each account probably obtains a superb quantity of on line foreign places coins as skip once more. In the second one section, the assaulter will device those remote places cash-collection bills to replace the digital cash to excellent payments. Numerous layers of shifting sports activities sports activities activities may be entailed to obfuscate the identities of malicious debts implemented for taking element on-line promo duties. At the save you of the second one degree, a huge amount of on line cash will certainly be collected into multiple laundering debts. In the zero. 33 phase, the assailant will control the laundering money owed to exchange the net coins into real coins with the beneficial resource of manner of the usage of advertising and marketing and advertising and marketing and marketing it to man or woman clients. Attackers generally make use of techniques to acquire character customers together with sending out spams and moreover advertising and marketing thru important purchasing internet internetinternet web web sites collectively with www.Tmall.Com. In order to compete with regulated property for online cash (i.E., trying to find virtual cash the usage of actual foreign places cash), enemies usually deliver a huge charge lessen. Our motive is to increase a discovery tool with the capability of identifying malicious payments that be a part of on-line promo sports 
sportssports for digital cash series (at the gathering diploma) in advance than rewards are committed. Spotting risky bills at this high-quality time factor (i.E., preceding to the strength of will of rewards and moreover at the gathering diploma) results in unique blessings. First, as a crucial heuristic to keep away from newly registered debts which may be likely to be bots, agency entities typically call for the taking detail bills to be signed up for a specific amount of time (e.G., some weeks). For that reason, the placed further to decreased negative debts can't be rapid changed through manner of way of the use of way of the freshly signed up money owed, therefore appreciably restricting assailants' abilities. On the other hand, no restraint is asked payments used for on-line coins transferringand laundering. This implies such payments can be outcomes modified thru manner of enemies if positioned, resulting negligible have an effect right now to attackers' abilities.

\section{EXPERIMENTAL REVIEW \& RESULTS}

We outline the tool studying techniques we have a propensity to notion of to deal with the hassle of figuring out mobile specific webpages as risky or benign. We typically will be predisposed to then talk the durability in addition to weaknesses of each classification technique, and moreover the method for deciding on the very amazing model for batter. We have a tendency to preserve together and determine our decided on version for accuracy, fake favorable price and true favorable charge. Ultimately, we commonly typically have a tendency to evaluation assault to offer techniques and moreover via experimentation show the significance of kAYO's options. We will be predisposed to be aware that any area automatic assessment is viable, we generally typically have a propensity to apply our whole datasets; but, as is usually damaged the assessment place, we will be predisposed to utilize arbitrarily decided on components of our facts as brief as large guide assessment and verification is wanted.

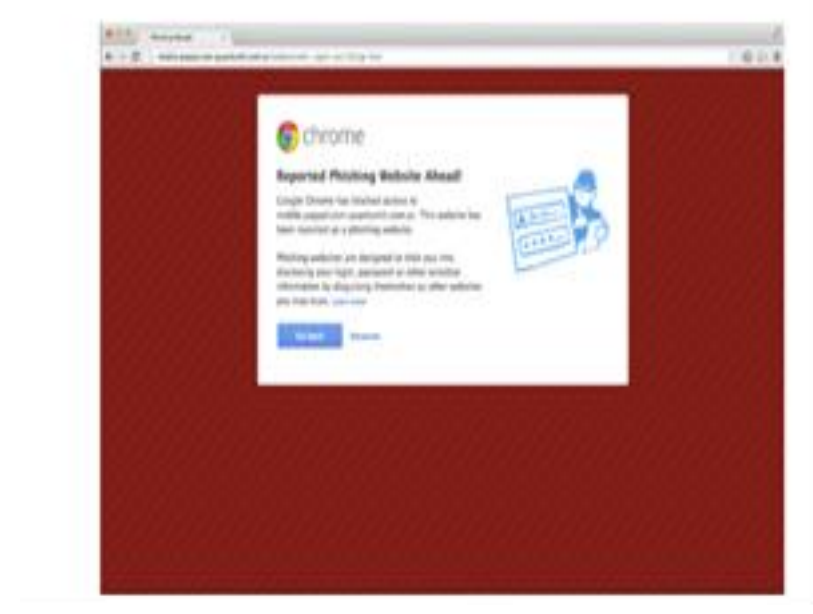

We usually typically will be inclined to treated draw bacthe hassle of detection malicious net net websites as a binary classification hassle. We have a tendency to concept of every well-known benign cellular internet internetinternet net page as an terrible sample similarly to each famous malicious cellular internet net website online on-line as a tremendous example. We will be predisposed to idea of a massive form of favored binary classification techniques in synthetic intelligence, apart from place talk approximately 3 properly-favored alternatives: Support Vector Machines (SVM), nave Bayes and moreover stipulation regression. Assistance Vector Machines (SVM) may be a well-favored binary classifier.However, it art work swell lonesome on more than one thousand samples of records. Way to the lowering detail of SVMs and our large dataset, SVM had not been the very first-rate preference for Nave Bayes is commonly made use of as rapid due to the reality the values of numerous alternatives place device reciprocally freelance. Several options that we will be predisposed to eliminated have been reciprocally reliant. For instance, the quantity of manuscripts in a truely internet internet net internet internet page turn out to be consumed on the amount of internal, outside similarly to ingrained JavaScript within the net net internet page, which have been 3 unique options of our version. Given that the assumptions desired for most fantastic overall performance of nave Bayes did not preserve for our dataset, we have a tendency to could not hire the naive Bayes classifier. Arrangement Regression may be a climbable classification method and makes no assumption touching at the go with the flow of worths of the options. For that purpose, this device have end up the very great fit for our dataset. We will be predisposed to made use of the binomial version of provision regression to model beat up and drastically completed '1-regularization to live smooth of overfitting of the records.We finished the scrapy net scratching form to transport slowly the collected cellular URLs. We commonly commonly usually have a propensity to then made a laptop software program software for extracting alternatives said in from every enter website dynamically. The crawler and feature extraction scripts were completed in Python. We will be inclined to used association regression on the drawn out alternatives for education similarly to locating out. We have a propensity to configured the stipulation regression version in the numerical computing language Octave we will be inclined to checked the version on an device with quad middle 3.Four fee Intel Core i7 processor and furthermore $16 \mathrm{~GB}$ memory.

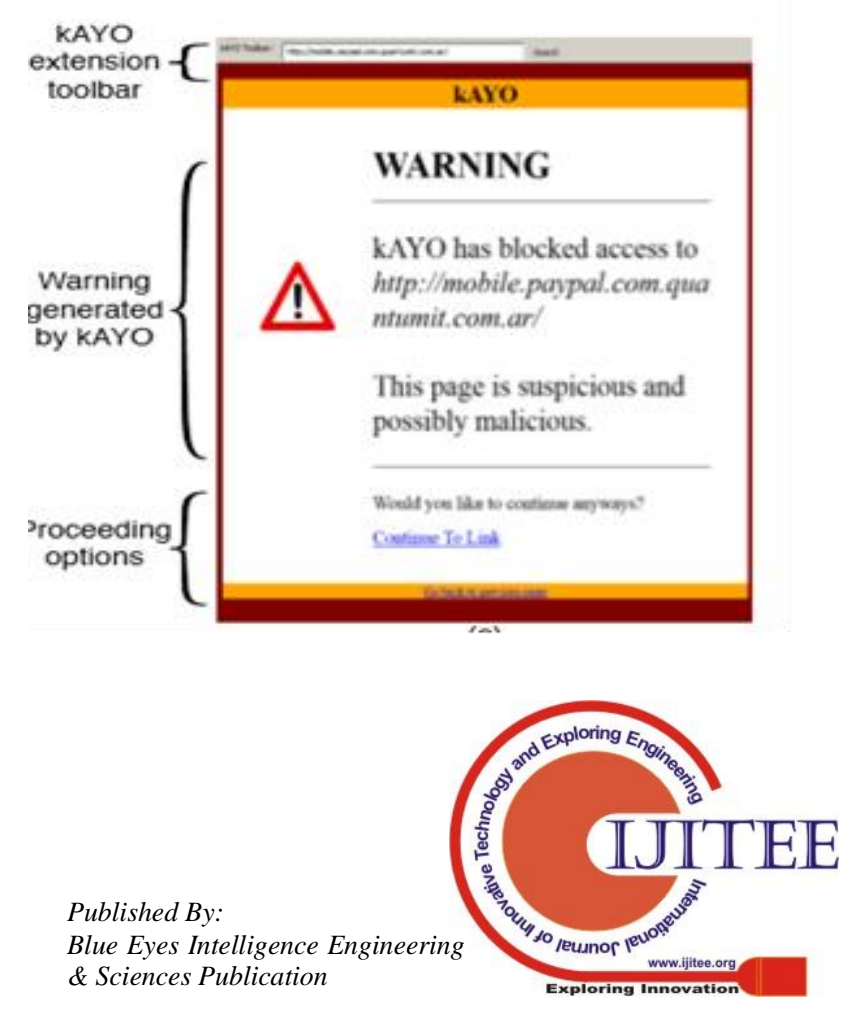




\section{CONCLUSION}

This paper offers an unique device, ProGuard, to routinely find out negative OSN bills that take part in online vending activities. ProGuard leverages 3 companies of attributes together with primary conduct, virtual-foreign places coins series, and moreover virtual-foreign exchange usage. Speculative results primarily based absolutely completely on labelled statistics accrued from Tencent QQ, a worldwide predominant OSN business organization, have confirmed the detection precision of ProGuard, which has in reality finished a immoderate detection price of ninety six.Sixty seven\% provided a really low incorrect favorable fee of $0.3 \%$.

\section{REFERENCES}

1. X. Hu, J. Tang, and also H. Liu, "Online social spammer detection,"in Process of the Twenty-Eighth AAAI Seminar on Expert System. AAAI, 2014, pp. 59-- 65.

2. "Leveraging knowledge throughout media for spammer detection in microblogging," in Process of the 37th international ACM SIGIR conference on Research study $\&$ advancement in info retrieval. ACM, 2014, pp. 547-556.

3. Z. Chu, S. Gianvecchio, H. Wang, as well as S. Jajodia, "Finding automation of twitter accounts: Are you a human, robot, or cyborg?" IEEE Deals on Reliable as well as Secure Computer, vol. 9, no. 6, pp. 811-824,2012 .

4. Z. Chu, S. Gianvecchio, A. Koehl, H. Wang, and also S. Jajodia, "Blog or block: Identifying blog bots via behavior biometrics," Computer system Networks, vol. 57, no. 3, pp. 634-- 646, 2013.

5. S. Fakhraei, J. Foulds, M. Shashanka, and also L. Getoor, "Cumulative spammer discovery in progressing multirelational social networks," in Procedures of the 21th ACM SIGKDD International Seminar on Understanding Exploration as well as Information Mining. ACM, 2015, pp. 1769-- 1778.

6. Y.-R. Chen and also H.-H.Chen, "Point of view spammer detection in web forum, "in Proceedings of the 38th International ACM SIGIR Conference on Research and Development in Details Retrieval.ACM, 2015, pp. 759-762.

7. F. Wu, J. Shu, Y. Huang, and also Z. Yuan, "Social spammer as well as spam message co-detection in microblogging with social context regularization,"in Proceedings of the 24th ACM International on Conference on Info as well as Understanding Management. ACM, 2015, pp. 1601-- 1610.

8. Z. Miller, B. Dickinson, W. Deitrick, W. Hu, and A. H. Wang, "Twitter spammer discovery making use of information stream clustering," Details Sciences, vol. 260, pp. 64-- 73, 2014.

9. H. Gao, J. Hu, C. Wilson, Z. Li, Y. Chen, as well as B. Y. Zhao, "Finding and characterizing social spam campaigns," in Process of the 10th ACM SIGCOMM seminar on Internet dimension. ACM, 2010, pp. 35-- 47.

10. S. Lee as well as J. Kim, "Warningbird: Finding suspicious links in twitter stream." in NDSS, vol. 12, 2012, pp. 1-- 13.

11. C. Yang, R. C. Harkreader, and also G. Gu, "Die free or online tough? Empirical evaluation and also brand-new design for combating developing twitter spammers," in International Workshop on Current Advancements in Invasion Discovery. Springer, 2011, pp. 318-- 337.

12. A. Abdallah, M. A. Maarof, as well as A. Zainal, "Fraud discovery system: A study," Journal of Network and also
Computer system Applications, vol. 68, pp. 90-- 113, 2016.

13. J. West and M. Bhattacharya, "Intelligent economic fraudulence detection: A detailed review," Computers \& Security, vol. 57, pp. 47-- 66, 2016.

14. D. Olszewski, "Scams discovery making use of selforganizing map envisioning the user accounts," Knowledge-Based Solution, vol. 70, pp. 324-- 334,2014.

15. C.-C. Lin, A.-A. Chiu, S. Y. Huang, and D. C. Yen, "Discovering the monetary statement fraudulence: The evaluation of the differences between data mining techniques as well as experts' judgments," KnowledgeBased Solution, vol. 89, pp. 459-- 470, 2015.

16. C. S. THROCKMORTON, W. J. MAYEW, M. VENKATACHALAM, AS WELL AS L. M.C. 